Voix et Images

\title{
Du sommet d'un arbre ou le regard en plongée et en quatre temps
}

\section{Renald Bérubé}

Volume 12, numéro 3 (36), printemps 1987

Yves Beauchemin

URI : https://id.erudit.org/iderudit/200655ar

DOI : https://doi.org/10.7202/200655ar

Aller au sommaire du numéro

Éditeur(s)

Université du Québec à Montréal

ISSN

0318-9201 (imprimé)

1705-933X (numérique)

Découvrir la revue

Citer cet article

Bérubé, R. (1987). Du sommet d'un arbre ou le regard en plongée et en quatre

temps. Voix et Images, 12(3), 404-415. https://doi.org/10.7202/200655ar d'utilisation que vous pouvez consulter en ligne.

https://apropos.erudit.org/fr/usagers/politique-dutilisation/ 


\title{
Du sommet d'un arbre ou le regard en plongée et en quatre temps
}

\author{
par Renald Bérubé, Université du Québec à Rimouski
}

à Yves Lacroix

Je cours même en dormant, me dit parfois ma mère avec un air de moquerie.

Du sommet d'un arbre, p. 22

Beauchemin n'écrira jamais que du Beauchemin, pour le meilleur ou pour le pire.

Ibid., p. 83.

Étrange métier que celui d'écrivain, que je n'arriverai jamais à comprendre tout à fait. La société, cette machine à fabriquer des rôles, m'en a donné un, merveilleux mais redoutable. Je sens mes vêtements trop grands qui flottent sur mon corps. Mon masque cherche à glisser. J'avance sur la scène, devant la salle silencieuse, en attente. On ne m'a pas donné de texte à réciter. On m'a seulement dit que la pièce doit continuer.

Ibid., p. 92.

Ce matin, je mets de côté pour quelques semaines la rédaction de mon roman et m'aventure pour la deuxième fois sur les pentes glacées de ce charmant casse-gueule appelé Journal intime, intime et radiophonique, discret comme une sonnerie de trompettes.

Ibid., p. 95.

Vous achevez, pages 138 et 139 , les deux dernières, votre lecture du plus récent livre d'Yves Beauchemin, Du sommet d'un arbre ${ }^{\text {, }}$, «journal» selon l'indication inscrite sur la couverture - jusque-là, vous aviez connu Beauchemin romancier, celui de l'Enfirouapé ${ }^{2}$ et du Matou ${ }^{3}$. Et le dernier passage du livre, presque irrésistiblement, vous rappelle son début et vous y renvoie, parcours bien connu des amateurs du circuit incipit-explicit (ou vice versa) - ce soir-là, à son bureau, à la veille de terminer son sprint dactylographique (p. 138), à la veille de mettre un point final au texte dont vous achevez la lecture, Beauchemin pense à ses enfants, Alexis l'aîné et Renaud: Depuis quelque temps, ma machine à écrire leur apparaît de plus en plus comme une ennemie sournoise qui leur vole des morceaux de père... (p. 138); et de recevoir bientôt, par l'émetteur-récepteur d'Alexis qu'il a dû apporter à la demande de ce dernier, le signal convenu (p. 139); message-demande d'Alexis: Papa... bonne nuit... Papa? [... ] Papa... n'oublie pas que je suis là, hein? (p. 139). Vous vous souvenez alors de la première phrase du Sommet: $M o n$ père travaillait pour les Américains (p. 13), et de cette autre, quelques pages 
plus loin, elle aussi située dans le premier des quatre textes du Sommet, celle où Beauchemin raconte sa propre enfance: ... sans mon père, souvent retenu par son travail (p. 29). Sorte de boucle bouclée, vous dites-vous, le circuit incipit-explicit, ici, traduisant à sa manière le parcours de la continuité du monde: nous naissons, enfants de nos parents, nous grandissons, et nos enfants nous rendent parents à notre tour, «le fils ou la fille de» devenant «le père ou la mère de" tout en restant l'enfant de ses origines. Spécularité et répétition, boucle: de Beauchemin fils évoquant son père à Beauchemin père qui a des fils, et quelque part dans la trajectoire, course ou sprint, continuité et spécularité, la mort, hantise et ancrage du Sommet, vous le savez, devra bien faire son nid et trouver sa place et son moment: Et surtout je songe avec tristesse que je suis passager dans un autobus qui ne fera le circuit qu'une fois avant de me laisser à ce Terminus où les voyageurs se transforment tout à coup en air pur (p. 89).

Mais il n'y a pas que le roman familial ou filial des êtres ou des personnages, il y a aussi celui des œuvres, qui n'est peut-être, après tout, que la version écrite ou manifeste, textuelle, du premier. Mais réfléchissant ainsi, vous savez bien que vous vous faites autobiographe à votre tour, le roman familial de vos lectures, chacune n'étant toujours qu'un élément du parcours de la bibliothèque borgesienne, entrant ici en scène et voulant jouer son jeu, offrir ses indices et ses pistes... de lecture. Donc: l'Enfirouapé et le Matou, romans, ont été lus d'abord, au moment de leur parution; lisant Du sommet d'un arbre, journal, c'est-à-dire, en l'occurrence, recueil de quatre textes, disons pour l'instant autobiographiques, d'abord destinés, à des moments différents, à la radio - lisant Du sommet d'un arbre, vous n'avez pu, vieil admirateur de la sagesse de Don Quichotte, l'ingénicux hidalgo, et pourquoi pas, empêcher votre lecture d'être marquée par l'un de ses bons mots: ...et chacun est fils de ses aeuvres ${ }^{4}$. Littéraire ou littéral jusqu'au bout, vous avez donc fait vôtre la formule du chevalier errant, la traduisant ainsi: Beauchemin est fils de l'Enfirouapé et du Matou - sans eux et leur succès, Du sommet d'un arbre nous eût-il jamais été donné à lire? La question prend ici les allures d'une réponse: non, probablement pas.

D'autant plus que, ingénieux quichottien à sa manière, Beauchemin s'autorise dans Du sommet, parlant de René Lévesque, de l'un des personnages de l'Enfirouapé:

J'approuve toujours le jugement qu'un des personnages de l'Enfirouapé portait sur lui: c'est le plus grand homme politique que le Québec ait connu depuis Jean Talon.

Retour à l'Enfirouapé, page 21, qui semble bien contenir le jugement dont s'autorise l'auteur du Sommet:

Et pourtant, non, il [ René Lévesque] ne ressemble pas tout à fait aux autres. Je ne sais pas pourquoi, il ne le sait peut-être pas lui-même, mais le fait demeure. À mon avis, c'est le premier politicien correct qui se montre la face chez nous depuis Jean Talon, rien de moins. 
Oui, oui, je sais, j’ai remarqué aussi: le personnage du roman parlait du premier politicien correct là où Beauchemin parle du plus grand homme politique - Beauchemin, sans doute, a droit à ses propres interprétations de ses propres romans, comme il a droit, ainsi qu'il l'écrit dans le Sommet, à l'éloignement de ceux-ci dans sa mémoire: Voilà une mèche que l'Enfirouapé est devenu pour moi comme une sorte de corps étranger et le Matou s'apprête à faire de même (p. 78). N'empêche que le Prince (Trudeau) du Sommet entretient bien des affinités avec le Prince de l'Enfirouapé; n'empêche que le je-Beauchemin du troisième extrait placé en épigraphe de ce texte entretient aussi des parentés avec le Gilles Pellerin de l'Enfirouapé - l'un et l'autre, agissant, agissent comme sur une vaste scène, et leurs agissements sont aussi tributaires de leurs lectures. Et comment, lisant ce (long) passage du Sommet où Beauchemin évoque son enfance rythmée par le passage assourdissant des longs convois de marchandises traînés par des locomotives à vapeur:

Trois heures du matin. Je dors profondément, mon chat roulé en boule à mes pieds, mes vêtements soigneusement rangés sur une chaise (une nouvelle habitude qui émerveille ma mère et la rend toute songeuse: les années passent, ses enfants changent). Soudain, un grondement sourd se fait entendre quelque part, très loin. Au bout d'une minute ou deux, la maison se met à vibrer doucement. J'ouvre un ceil. Mon frère se retourne dans son lit en soupirant. Le grondement ne cesse de grossir et se rapproche de plus en plus. Il roule maintenant comme une avalanche. Et tout à coup, un hurlement déchirant éclate dans la nuit tandis qu'une longue file de wagons se rue à travers le village dans un vacarme féroce. Je regarde par la fenêtre. La lune essaie de percer une grosse masse de nuages bleu sombre au-dessus du lac. Le hurlement retentit de nouveau. On dirait une grande bête frappée à mort. Je sens tout autour de la maison une immensité froide et noire, sans limites, où rôde le malheur. Les murs de la maison me semblent minces comme du papier.

comment ne pas se souvenir de cet autre passage, lu bien avant, du Matou:

Vers trois heures du matin, un sifflement les réveilla brusquement. Quelques instants passèrent. Le sifflement résonna de nouveau, plus rapproché. - Ma foi! c'est un train murmura Élise en se dressant dans le lit.

Un grondement sourd se répandit peu à peu dans le sol gelé et monta dans les murs. Florent se précipita à la fenêtre. On ne voyait pas à dix pieds. Sans crier gare, l'hiver avait sorti sa faux et fendu tous les nuages. Des tonnes de neige tombaient doucement du ciel, plongeant le pays dans une atmosphère solennelle et mystérieuse. Le grondement augmentait toujours et se transforma soudain en tonnerre. Les vitres se mirent à tinter et Vertu [ la chienne ], au comble de la désolation, poussa un hurlement lugubre. Elise fut prise d'une peur subite, irraisonnée. Elle courut 
rejoindre son mari et lui cria quelque chose, mais il ne l'entendait pas: possédée par toutes les fureurs de l'enfer, une grosse masse noire filait devant la gare dans un fracas étourdissant.

(Le Matou, p. 413)

Parentés nombreuses et précises: "trois heures du matin", "grondement", l'"avalanche" métaphorique et les "tonnes de neige» réelles, la fenêtre par laquelle on regarde, le «malheur", le "fracas", l'«enfer». Or le Matou, roman, est une œuvre de fiction et le Sommet, journal, une œuvre à caractère autobiographique - "sans crier gare», pour rester dans le registre ludique du Matou, Beauchemin, décrivant une scène répétitive de son enfance, dans le Sommet, et une situation inattendue, deus ex machina, dans le Matou, décrit la même scène ou à peu près, leurs parentés textuelles témoignant bien de leur proximité dans la mémoire-imagination. Alors, Quichotte l'ingénieux? Si Beauchemin, fils de ses œuvres, est bien le fils de l'Enfirouapé et du Matou, ses romans et ses œuvres, ses parents donc, de quoi s'agit-il s'agissant du Sommet dont il est l'auteur et l'actant principal, du Sommet qui n'a sans doute vu le jour que grâce aux vertus successful des romans antérieurs, et qui relate tout à la fois l'origine et le présent de l'auteur? Des origines et du présểnt du fils des œuvres, nous répondrez-vous sans doute, ingénieux et logique, c'est-à-dire astucieux et rusé, retors et lecteur attentif - vous nous direz; ce faisant, freudien avant la lettre et avant Marthe Robert ${ }^{5}$ utilisant les acquis de Freud - souvent évoqué par Beauchemin dans le Sommet - pour parler de vous, Don Quichotte ou Don Quichotte, que l'ancien et le nouveau sont intimément liés, que nous sommes aussi les fruits, fils et filles, de notre archéologie, que l'origine du roman n'est toujours que le roman des origines. $\mathrm{Ou}$, lecteur attentif et borgesien, vous citerez, nous ramenant à nos préoccupations immédiates, l'auteur du Sommet: Beauchemin n'écrira jamais que du Beauchemin, pour le meilleur ou pour le pire (p. 83).

S'agit alors de savoir ce qui s'écrit et comment cela s'écrit.

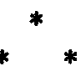

Du sommet d'un arbre, journal, a donc été publié en 1986. Le livre se divise en quatre parties: «Enfance» (p. 11-34), «la Ville» (p. 35-46), «Journal 1983 " (p. 47-92), «Journal 1985" (p. 93-139). Les deux dernières parties, leurs titres l'indiquent assez clairement, se présentent bien sous la forme du journal: datées du 22 février au 10 mai 1983, puis du 12 février au 17 avril 1985, elles sont composées de textes plus ou moins longs, portant sur des sujets divers, textes délimités par les dates, comprises entre celles du début et de la fin, auxquelles ils ont été écrits. Ces deux journaux occupent les deux tiers environ du recueil, ce qui peut expliquer la dénomination générique donnée au Sommet. Les parties une et deux, quant à elles, la première surtout, sont de nature plus proprement autobiographique et peuvent assez bien se lire selon la définition de l'autobiographie proposée par Lejeune: 
Récit rétrospectif en prose qu'une personne réelle fait de sa propre existence, lorsqu'elle met l'accent sur sa vie individuelle, en particulier sur l'histoire de sa personnalitét.

L'auteur, le narrateur et le personnage principal sont bel et bien ici la même personne et la perspective est celle de la rétrospection. Et l'incipit de ala Ville»: Ainsi donc, je devins... (p. 37) insiste sur le lien qui l'unit à «Enfance": chronologiquement, les événements relatés dans «la Ville» s’inscrivent dans la continuité de ceux racontés dans "Enfance». Pourtant, les titres mêmes des deux parties soulignent aussi une rupture: là où «Enfance» renvoie à un moment de la vie personnelle, "la Ville» signale un lieu, le lieu où et par lequel adviendra une nouvelle étape de la vie personnelle, qui marquera la fin de la première enfance. On a même l'impression, lisant "la Ville" immédiatement après «Enfance», que le lieu, Montréal, devient ici, tout autant que le je, personnage principal du texte. Cela étant, il faut alors revenir en arrière et relire les indications placées tout au début du Sommet:

Les textes du présent recueil ont été écrits pour le réseau CBF-FM de Radio-Canada. Enfance a été diffusé le 17 janvier 1979 sous le titre Un enfant de village dans la série Un écrivain et son pays (réalisation: Louis-Philippe Hébert). La Ville a été diffusé le 30 avril 1985 dans la série Eloges (réalisation: Aline Legrand). Journal 1983 et Journal 1985 ont été diffusés respectivement du 15 au 19 août 1983 et du 10 au 14 juin 1985 dans la série Journal intime (réalisation: Jean Lacroix).

Le moins qu'on puisse dire: renseignements génétiques fort nombreux et tout aussi fascinants. "La Ville» a donc été diffusé dans la série Éloges (de?): ce qui pourrait rendre compte de l'importance qu'y prend Montréal, de l'espèce de célébration qu'en fait le je? Mais il n'y a pas que ce renseignement sur l'optique dans laquelle a pu être conçue «la Ville»; «Enfance»", dans son titre premier, mettait l'accent à la fois sur un être et un lieu - et ce texte a été diffusé avant la parution du Matou: qu'en est-il alors de ces deux extraits sur "le passage du train» que nous citions tout à l'heure? L'un est-il bien antérieur à l'autre, et si oui lequel? Et si nous lisons, respectant l'ordre du Sommet, "la Ville» avant "Journal 1983 », nous lisons avant un texte qui a été diffusé après. Du sommet d'un arbre est donc bien un recueil, un montage de textes organisé selon l'ordre chronologique du contenu de ces textes; textes forcément remaniés et réaménagés par rapport à leurs avant-textes, pour utiliser la terminologie de Jean Bellemin-Noël ${ }^{8}$, avant-textes à destinataire radiophonique. Lorsque nous lisons, dans « Journal 1983»: il s'agissait donc, lorsque j'ai commencé la rédaction de ces cinq demi-heures... (p.81), ou dans "Journal 1985": ... content tout de même du voyage intérieur que ces cinq textes m'ont permis de faire, malgré tous leurs défauts (p. 138), force nous est de constater que ces divisions radiophoniques en cinq sont absentes des journaux publiés. Retour à Lejeune: 
Dans les années 1950, l'autobiographie orale s'est développée à partir de situation [ sic ] orales réelles, celles des entretiens destinés à la radio: la transcription de l'oral à l'écrit était alors un phénomène secondaire. Ce qui caractérise le développement intensif de l'autobiographie orale ces dernières années, c'est au contraire que le processus oral est déclenché dès l'origine pour produire un livre; le public n'aura jamais été ni témoin, ni garant de l'oralité initiale, et ne saura pas toujours très bien comment a été fabriqué le texte qu'il lit ${ }^{9}$.

Citation qui prend ici les allures d'un prétexte: prétexte à souligner les problèmes réels qu'elle soulève, prétexte à souligner surtout ce qui distingue $\mathbf{D u}$ Sommet d'un arbre des situations évoquées par Lejeune: 1. je ne sais si, au moment d'écrire «Un enfant de village», Beauchemin pensait déjà en termes de livre; 2. destinés primitivement à la radio, les textes du Sommet n'en furent pas moins au départ des textes écrits et non des entretiens oraux; 3 . les indications données, au début du livre, sont comme une invite à confronter textes et avant-textes, signalant comment le livre a été fabriqué.

Résumons-nous: Du sommet d'un arbre, journal, est constitué d'une première partie proprement autobiographique, d'une deuxième en forme d'séloge» de Montréal prolongeant l'autobiographie, et de deux journaux. Quatre moments dans la vie d'Yves Beauchemin. Si bien lancés dans la lecture des sous-titres et autres éléments paratextuels ${ }^{10}$, peut-être devrionsnous nous attarder sur le titre - pourquoi Du sommet d'un arbre? Et risquer les remarques suivantes, qui vont nous obliger à quelques détours, aériens et autres.

Tout au début du "Journal 1983", Beauchemin évoque (il avait alors 8 ou 9 ans - p. 49) le moment où il a entendu pour la première fois la Cinquième de Beethoven - il se souvient même de l'illustration ornant le gros album à couverture cartonnée (p. 49): Beethoven, l'air sinistre, se promenait courbé dans la tempête, les mains ramenées dans le dos. Derrière lui, la foudre venait de frapper un arbre noir et dénudé sur le haut d'une colline (p. 49); mais surtout, il se souvient de l'effet produit par le deuxième mouvement de la Cinquième:

Jamais je n'avais rien senti de pareil. Je connaissais le plaisir de la lecture. Mais il s'agit d'un plaisir lent, qui se construit page après page en nous, indifférent à nos yeux affamés qui voudraient dévorer le livre d'un coup. Tandis que là... c'était l'extase... l'impression de posséder la terre et les étoiles, de dépasser le temps... [...]

$[\ldots]$

En fait, j'attendais que le fameux thème de la marche revienne et me refasse son effet d'hélicoptère. Il finit par revenir. Je montai de nouveau dans l'espace. C'était ça. À côté de ça, il n'y avait pas grand-chose dans la vie qui importait vraiment. 
Les causes de l'effet d'hélicoptère peuvent être multiples et varier selon les individus : Et mon éventail à moi est-il bien large? En cherchant un peu, je trouve péle-mêle: l'amour, la musique, la lecture, un bon film, le jogging (ce fameux "orgasme d'oxygène» qui donne l'impression de pouvoir courir indéfiniment sans fatigue) (p. 54). Imprévisible quant au(x) moment(s) de sa manifestation, les mêmes causes ne produisant pas toujours le même effet, l'effet d'hélicoptère peut, exemplairement et suite à ce qui vient d'en être dit, se résumer et se définir selon le passage suivant:

L'effet d'hélicoptère est capricieux. Il fond sur nous par surprise. J'ai fait une envolée sublime il y a plusieurs années en entendant, grâce à une méchante petite radio, la Neuvième de Mahler dirigée par Sir John Barbirolli. L'extase, les larmes et tout. Jamais personne ne m'avait parlé d'une façon à la fois si triste et si consolante de la mort, de ma mort. Deux ans plus tard, j'achète l'enregistrement de la symphonie, je pose le disque sur ma platine, j'enfile mon habit de vol et je m'assois. L'hélicoptère n'a jamais décollé.

Tout à la fois «extase», "envolée» et «orgasme», l'effet d'hélicoptère foudroie et propulse vers le(s) sommet(s); il arrache l'être à lui-même, lui donne (lui rend?) le sentiment de la (sa?) toute-puissance: dépasser le temps, pouvoir courir indéfiniment sans fatigue. Arrachement, envol ou extase, qui n'est toujours, paradoxe, qu'une façon de mieux cerner sa place dans le temps et le monde, de mieux coïncider avec soi-même: la Neuvième de Mahler parle à Beauchemin de sa mort, il s'écoute l'écoutant, il se (re)trouve s'envolant. L'effet d'hélicoptère permet le regard en plongée, à la fois panoramique et centré; il permet de faire le point ou de marquer un point, de ramasser l'éparpillé - ce que peut permettre aussi le journal? Grâce à la Cinquième et à son effet d'hélicoptère, monsieur Beethoven de Vienne [...] racontait quelque chose d'infiniment simple et complexe à la fois, qui avait figé sur place dans son village d'Abitibi un petit garçon de 8 ans aux souliers remplis de sable (p. 50) - il avait, de fait, aboli temps et distance, la mort, permis la continuité (lire aussi à ce sujet, continuité du Sommet, à la page 128). Arbre foudroyé, l'auditeur Beauchemin n'en était pas moins en pleine envolée - Yves Beauchemin, le petit garçon caché dans un adulte qui vient de s'acheter un walkman pour connâtre un peu plus souvent l'effet d'hélicoptère (p. 50) tente la même entreprise, écrivant son journal du sommet d'un arbre (je ne sais s'il faut, ici, souligner les derniers mots)?

Dernière remarque sur l'effet susdit: il permet de découvrir le monde de la même façon que Balzac (Beauchemin parle aussi de l'effet Balzac, p. 41) permet à l'étudiant Beauchemin, par le cours d'Ernest Gagnon, de découvrir Paris (p.67-68). 
Il est peut-être arrivé, écrivant les pages qui précèdent et qui renvoient à un lecteur ayant son parcours et ses bibittes, c'est-à-dire une (auto)biographie, que j'aie omis d'écrire ceci: Du sommet d'un arbre est un petit livre chaleureux et attachant, passionnant et simple - d'une simplicité redoutable pour employer cet adjectif selon Beauchemin (p. 76, 80, 81, 89, 92, 115). Conteur, fils de monsieur Émile l'ingénieux, Beauchemin raconte, et en accéléré net plutôt qu'au ralenti contourné: Du sommet, selon la formule consacrée, se lit tout d'une traite; mais il faut ensuite le relire au ralenti. De même pour l'Enfirouapé et le Matou: l'agencement, l'emballement si minutieusement organisé des intrigues, aventures, événements et autres rebondissements multiples, d'une remarquable efficacité appuyée aussi par l'humour, ne doit pas gommer sa propre minutie, son perfectionnisme (p. 89), simplicité "redoutable» l'emballement des intrigues, aventures, etc., n'est toujours que la traduction, à décoder, de la complexité des personnages, par exemple. Beauchemin, romancier ou diariste, n'explique pas, il raconte; mais nous savons, astucieux et ingénieux, et Beauchemin le sait aussi (p. 81), que la façon de raconter n'est jamais innocente, tissant ses explications dans son tracé même.

Dans le Sommet, Beauchemin raconte donc son enfance à Clova, en Abitibi, petit village québécois qui appartenait aux États-Unis (p. 21), sa venue hâtive et forcée à la lecture-écriture (...refoulé dans le monde de l'imagination, p. 20), sa découverte émerveillée de Montréal (La ville, ce gros livre..., p. 43) et de sa condition de Québécois (p. 43); dans les journaux, genre oblige, Beauchemin aborde divers sujets, les abandonne pour mieux y revenir par un autre biais, tramant, dans l'apparente discontinuité et mine de rien, une réflexion beaucoup plus poussée que ses dehors souvent amusés ne pourraient le laisser croire. S'il parle encore un peu de son enfance, Beauchemin y parle surtout de ses enfants, du drame que fut pour eux la mort du poisson rouge, par exemple (p. 71-72), nous racontant même au passage, conteur impénitent, l'histoire des histoires qu'il leur raconte (p. 104-107 et 110-113) et nous faisant part de ce fait, critique attentif, de leurs réactions. Famille encore, mais plus vaste, la québécoise: la question nationale occupe une large place dans les journaux, Beauchemin y discutant des années de pouvoir du Parti québécois, mon pays [ qui] semble parti à la dérive (p. 132), et du fait à la fois inéluctable et malheureux que l'indépendance est passée de mode (p. 61). Tout passe, constatation qui hante Beauchemin - Homère et Virgile, déjà, ne sont plus audibles, pour la vaste majorité des lecteurs, que par le tuyau plein de fuites des traductions (p. 129).

Car Beauchemin, on s'en doutait bien un peu, nous entretient aussi, dans Du sommet, de ses lectures et de son métier d'écrivain, drôle de métier [...] cette auto-psychanalyse pour diffusion publique (p. 73), métier parfois si redoutable (p. 81). Difficulté des incipits, sortes "de saut dans le vide" même si l'auteur a essayé de tout prévoir (p. 52), choix délicat du nom des personnages (p. 51), pièges de l'écriture autobiographique (p. 80-82 et 95-96) - et quelles différences, qui n'en sont pas tout à fait, peuvent bien distinguer l'autobiographie et le roman du point de vue de l'écrivain (p. 81-82)? Beauchemin raconte, joue avec le langage, discute avec lui-même: des dangers du 
succès et de la mythification, de l'avenir de son œuvre (p. 78-80). De la musique aussi, sa passion, source des plus merveilleux effets d'hélicoptère; de l'amitié, de la mémoire du cour (p. 139).

Bien sûr (?), on aura compris que ce résumé des sujets abordés dans le Sommet, que ce regroupement de quelques sujets abordés dans le Sommet n'est lui-même pas innocent 11 - il a ses intentions et sa direction, fruits, bien sûr, de la lecture du livre ou du recueil, journal. Enfant, Beauchemin avait appris [ $d$ ] ebout à l'arrière de la camionnette Ford qui filait sur le chemin raboteux, tout en tortillages, [...] les rapports mystérieux entre les parfums et la vitesse. $\dot{A}$ quarante milles à l'heure, la senteur des sapins fait tourner la tête, donne des idées loufoques et développe extraordinairement l'appétit (p. 18); et sa mère lui disait, avec moquerie, qu'il courait même en dormant (p. 22). Plus âgé et devenu romancier, Beauchemin écrit son premier jet à bride abattue, cherchant la griserie (p. 53); et nous dit-il encore, je me rappelle aussi quelques envolées dans le ciel de la fiction où je reste cloué durant des heures devant le tableau de bord de ma vieille Olivetti, le temps aboli, mon âme multipliée par trois, cinq, dix personnages, les difficultés de la langue jetées dédaigneusement par-dessus l'épaule, fonçant furieusement dans mon histoire pour $y$ chercher mon souffle de vie... (p. 73) - tout n'étant qu'éphémère, tout passant vite et le voyageur connaissant le Terminus (p. 89), l'écriture cherchera donc, accélérée, à prendre le temps de vitesse, le doublant et le dépassant, ultime effet d'hélicoptère rêvé, reste absolu du rêve archaïque de toute puissance. Mais l'écriture ne sait si bien créer l'illusoire que parce qu'elle (re)connaît l'illusion; et pourtant, des traces demeurant et la mémoire (du coeur ou autre) étant cultivée, faces duelles d'une même nécessité, René Lévesque continuera Jean Talon, et Yves Beauchemin, ravi et à sa manière, saura poursuivre Beethoven en l'écoutant et le faisant connaître. L'enfant et le parent, ne font qu'un, de même que l'individu et la collectivité - lire ici l'extrait suivant d'un texte de Beauchemin récemment publié dans Liberty(é):

Je suis écrivain. Mais je suis aussi un Québécois, père de deux enfants. L'aventure collective que nous vivons en Amérique depuis plus de trois siècles, commencée avec mes ancêtres, me traverse et se poursuit dans la vie de mes deux fils et des enfants qu'ils auront peut-être. Au-dessus de tout calcul et de tout raisonnement, je souhaite passionnément que cette aventure se continue dans les meilleures conditions possibles. D'une certaine façon, l'avenir du français au Québec se confond avec l'avenir de mes deux fils, avec l'avenir de tous les enfants québécois. Je ne peux vraiment pas rester indifférent aux forces qui d'une façon ou d'une autre tendent à infléchir la trajectoire de cette aventure pour lamener dans le cul-de-sac de l'assimilation et de la mort culturelle ${ }^{12}$.

Voilà bien, sur le mode de l'essai politique engagé, une sorte de résumé de Beauchemin par Beauchemin lui-même: lutter contre le Terminus, assurer la continuité et la pérennité des groupes et des individus. La langue est le lieu qu'habite l'écrivain; Québécois qui a ses ancêtres, de Jean Talon à René 
Lévesque, Yves Beauchemin veut assurer, pour et par ses fils, le moment de la continuité et de la collectivité qu'il constitue et qu'ils constitueront à leur tour. Ce qui explique sans doute que Beauchemin romancier, tout au début de l'Enfirouapé, ait, dans une sorte de prologue de moins de deux pages, résumé l'histoire de la famille du héros depuis le 16 octobre 1665, date de l'arrivée à Québec de l'ancêtre français, Rodrigue Ferland de la Barre, jusqu'au présent, 1970, du début de la narration des aventures du personnage principal, Maurice Ferland, le nom de famille originel ayant été ainsi réduit lors de [ $l$ ]'arrivée des Anglais (l'Enfirouapé, p. 5). D'ailleurs, le Sommet pourrait bien se lire tout entier dans l'un de ses propres extraits: retourner à ce passage du "Journal 1985 », page 98 , daté du 13 février. Beauchemin y raconte la visite que lui et son collègue Bruno Roy viennent de faire d'une ancienne école désaffectée en vue de son achat et de sa réfection éventuels par l'UNEQ qui caresse un vieux rêve: l'existence d'une Maison des écrivains. Récit étrange et émouvant, humoristique et fort sérieux, qui ramène Beauchemin à sa naissance et l'oblige à réfléchir à l'irréversibilité du temps (p. 102); et qui se terminera chez le disquaire Archambault (p. 103). Texte superbe, sorte de mise en abyme ou de récit dans le récit si cela pouvait se dire d'un journal et qui pourrait, exemplaire, illustrer Beauchemin diariste et romancier.

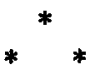

Beauchemin, donc, n'écrira jamais que du Beauchemin. Et vous vous dites, votre (première) lecture maintenant achevée depuis un moment, que le Sommet est un livre important; non seulement en lui-même, mais en regard de nouvelles lectures de l'Enfirouapé et du Matou - comment ne pas tenir compte des parentés de l'autobiographe/diariste et du romancier et des explications qu'il tente lui-même d'en donner? Ou encore, de la manière Beauchemin d'habiter le monde: le jeune lecteur de Clova, le refoulé dans le monde de l'imagination qui n'avait sa place dans les jeux que quand il y avait des choses à inventer (p. 20) parlera plus tard de [ $l]$ a ville, ce gros livre (p. 43); et lisant Chandler, il lira aussi comme chandlerienne telle situation dans laquelle il se trouve au Parlement, à Québec (p. 119-120). Le XVIe siècle, Cervantès et Shakespeare doivent bien s'en souvenir, parlait du théâtre du monde; traduisant selon le genre chéri du $\mathrm{XX}^{\mathrm{e}}$, le roman, nous pourrions dire que Beauchemin habite, c'est-à-dire lit, le monde comme un roman, le roman du monde. Sauf que sur la ... scène du roman, il est auteur tout autant que lecteur: J'avance sur la scène, devant la salle silencieuse, en attente. On ne m'a pas donné de texte à réciter. On m'a seulement dit que la pièce doit continuer (p.92).

Lectures s'entrechoquant, intertextualité, vous vous dites qu'il faudrait évoquer, à l'occasion du Sommet, d'«Enfance" plus particulièrement, tel et tel passage (du train?) de Rue Deschambault ${ }^{13}$ de Gabrielle Roy; tel passage précis de Lumière d'août ${ }^{14}$ de William Faulkner soulignant l'importance déterminante que peut revêtir le nom donné aux personnages; évoquer enfin le Coeur est un chasseur solitaire ${ }^{15}$ de Carson McCullers, à cause du rôle 
capital que la musique... joue dans la vie de la jeune Mick Kelly. Mais ce sera pour une prochaine ou d'autres lectures, pour un autre moment du journal des lectures. Car le Terminus, Beauchemin le sait bien, ce peut aussi être l'échéance, la date de tombée d'un texte. Même que l'autobus, je le sais, attend depuis un moment.

1. Yves Beauchemin, Du sommet d'un arbre, Montréal, Québec/Amérique, «Littérature d'Amérique", 1986. Dorénavant, s’agissant de ce livre, nous donnerons la référence immédiatement après la citation.

2. Yves Beauchemin, l'Enfirouapé, Montréal, la Presse, "Écrivains des deux mondes», 1974.

3. Yves Beauchemin, le Matou, Montréal, Québec/Amérique, "Littérature d'Amérique», 1981.

4. Cervantès, Don Quichotte de la Manche, tome I, Verviers (Belgique), Marabout géant illustré, Gi 4, s.d., p. 41.

5. Marthe Robert, l'Ancien et le nouveau (De Don Quichotte à Kafka), Paris, Payot, PBP no 105, 1967; Roman des origines et origines du roman, Paris, Gallimard, «Tel" no $13,1976$.

6. Philippe Lejeune, le Pacte autobiographique, Paris, Seuil, «Poétique», 1985, p. 14.

7. Titre qui pourrait avoir été influencé par Tolstoï? Je n'ai jamais abandonné de roman. Pardon, oui, une fois, mon premier, écrit à l'approche de la vingtaine pendant mes années de collège. [...] Je conserve le manuscrit, que je n'ai pas relu depuis dix-huit ans et qui doit être passablement influencé par Enfance de Tolstoï, un livre qui m'avait marqué à l'époque. (Du sommet d'un arbre, p. 82-83)

8. Jean Bellemin-Noël, le Texte et l'avant-texte, Paris, Larousse, «L», 1972.

9. Philippe Lejeune, op. cit., p. 338, note 2 .

10. Gérard Genette définit ainsi le paratexte:

Le second type [de relations transtextuelles] est constitué par la relation, généralement moins explicite et plus distante, que, dans l'ensemble formé par une ceuvre littéraire, le texte proprement dit entretient avec ce que l'on ne peut guère nommer que son paratexte: titre, soustitre, intertitres; préfaces, postfaces, avertissements, avant-propos, etc.; notes marginales, infrapaginales, terminales; épigraphes; illustrations; prière d'insérer, bande, jaquette, et bien d'autres types de signaux accessoires, autographes ou allographes, qui procurent au texte un entourage (variable) et parfois un commentaire, officiel ou officieux, dont le lecteur le plus puriste et le moins porté à l'érudition externe ne peut pas toujours disposer aussi facilement qu'il le voudrait et le prétend. (Pailimpsestes. La Littérature au second degré, Paris, Seuil, "Poétique», 1982, p. 9).

Au moment d'écrire cet article, parait, du même Gérard Genette, une étude entièrement consacrée au paratexte: Seuils, Paris, Seuil, "Poétique», 1987. À lire, bien sûr. Lectures, lectures...

11. Pas plus ni moins, sans doute, que la prière d'insérer suivant accompagnant le Sommet:

Vous connaissez le jeune coq-l'oeil de Clova? Non? Alors, Du sommet d'un arbre vous racontera non seulement une partie de l'enfance de Yves Beauchemin mais ses années d'apprentissage à Montréal pendant qu'il était étudiant à l'Université de Montréal puis des événements cueillis au jour le jour dans les deux «journaux intimes" (1983 et 1985) qu'il a écrits pour la Radio MF de Radio-Canada. L'auteur du Matou se raconte sans complaisance, avec la franchise qui le caractérise. Une percée dans la vie familiale des Beauchemin, une visite de sa maison à Saint- 
Lambert ( $y$ inclus les fondations!). Des morceaux de vie intime mais aussi des réflexions sur la politique, le mouvement indépendantiste, la ville de Montréal (qu'il faut sauver de la laideur). Des réflexions sur l'écriture et la gloire... Quelques intantanés [sic] pour mieux saisir sur le vif le plus célèbre de nos auteurs québécois.

Ou encore que la présentation suivante apparaissant sur la quatrième de couverture du livre:

Quand on porte des lunettes aux verres épais comme ça, qu'on est malhabile comme c'est pas possible, c'est-à-dire absolument incapable de frapper la balle comme du monde ou de lancer la rondelle du bon côté, il est normal qu'on veuille se venger d'être né gaucher. Alors on se tourne vers les livres et on empoigne son crayon à mine comme une épée. Gare aux mots!

Yves Beauchemin est devenu écrivain très tôt à Clova, en Abitibi. Plus tard, comme la plupart de ses confrères inscrits en Lettres, il a rêvé de gloire. Mais quand fut publié, [ sic ] Le Matou, personne ne pouvait prévoir que ce roman battrait le record de tous les temps. Plus d'un million d'exemplaires! Traduit en une dizaine de langues. De quoi lui enfler la tête. Mais l'homme est resté simple, humble même. Et profondément québécois.

Nous nous attachons $\dot{a}$ Yves Beauchemin parce que Beauchemin c'est, pour beaucoup d'entre nous, le rêve de ce que nous voulions être...

Une légende, un conte de fée.

Le prière d'insérer doit être allographe, car Beauchemin n'aurait certes pas confondu le Longueuil qu'il habite, et dont il est question dans le Sommet, avec Saint-Lambert. Les deux textes, par ailleurs, produisent le même effet de familiarisation dirait Genette (Palimpsestes, p. 69), et jouent sur la mythologie du "Vécu», ajouterait Lejeune (Le Pacte autobiographique, p. 339, note 1). Et jouent aussi sur nos rêves et nos fantasmes, dont nous espérons tous, bien sûr, qu'ils seront un jour bénis par les fées.

12. Yves Beauchemin, "l'Affichage français", dans Liberty(é), numéro non numéroté c'est-à-dire hors série, février 1987, p. 37-43. Titre de ce... numéro: Watch ta langue! Et une note au bas de la première page de l'article de Beauchemin fournit les renseignements suivants: "Mémoire (légèrement remanié, toujours d'actualité) présenté à la Commission élue permanente des Communautés culturelles et de l'immigration le 7 octobre 1983 ». Mémoire d'abord écrit, donc, après le “Journal 1983», et présenté avant la diffusion radiophonique de "la Ville" qui précède de peu celle de "Journal 1985".

13. Gabrielle Roy, Rue Deschambault, Montréal, Beauchemin (!), 1960.

14. William Faulkner, Lumière d'août. le Livre de poche, no 753-755, 1966, p. 53-54 pour le "passage précis".

15. Carson McCullers, le Coeur est un chasseur solitaire, le Livre de poche, "Biblio", no 3025. 1985.

Et sera-t-il permis d'ajouter, à l'occasion de cette note, la dernière, que les souvenirs que je garde du cours donné par Ernest Gagnon sur Balzac coïncident assez bien avec ceux de Beauchemin? Lectures, lectures, souvenirs, souvenirs... 